PREPARED FOR THE U.S. DEPARTMENT OF ENERGY, UNDER CONTRACT DE-AC02-76CH03073

PPPL-3833

PPPL-3833

UC-70

Nonlinear Simulation Studies of Tokamaks and STs

by

W. Park, J. Breslau, J. Chen, G.Y. Fu, S.C. Jardin,

S. Klasky, J. Menard, A. Pletzer, B.C. Stratton,

D. Stutman, H.R. Strauss, and L.E. Sugiyama

July 2003

NM|

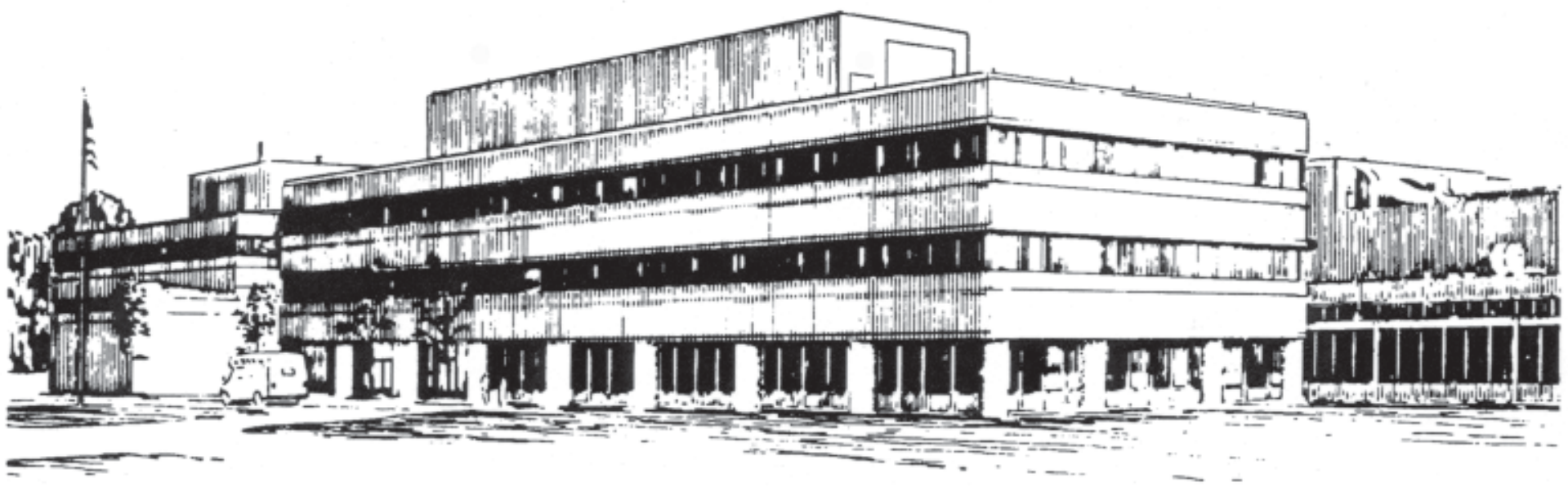

PRINCETON PLASMA PHYSICS LABORATORY PRINCETON UNIVERSITY, PRINCETON, NEW JERSEY 


\section{PPPL Reports Disclaimer}

This report was prepared as an account of work sponsored by an agency of the United States Government. Neither the United States Government nor any agency thereof, nor any of their employees, makes any warranty, express or implied, or assumes any legal liability or responsibility for the accuracy, completeness, or usefulness of any information, apparatus, product, or process disclosed, or represents that its use would not infringe privately owned rights. Reference herein to any specific commercial product, process, or service by trade name, trademark, manufacturer, or otherwise, does not necessarily constitute or imply its endorsement, recommendation, or favoring by the United States Government or any agency thereof. The views and opinions of authors expressed herein do not necessarily state or reflect those of the United States Government or any agency thereof.

\section{Availability}

This report is posted on the U.S. Department of Energy's Princeton Plasma Physics Laboratory Publications and Reports web site in Fiscal Year 2003. The home page for PPPL Reports and Publications is: http://www.pppl.gov/pub_report/

DOE and DOE Contractors can obtain copies of this report from:

U.S. Department of Energy

Office of Scientific and Technical Information

DOE Technical Information Services (DTIS)

P.O. Box 62

Oak Ridge, TN 37831

Telephone: (865) 576-8401

Fax: (865) 576-5728

Email: reports@adonis.osti.gov

This report is available to the general public from:

National Technical Information Service

U.S. Department of Commerce

5285 Port Royal Road

Springfield, VA 22161

Telephone: $1-800-553-6847$ or

(703) $605-6000$

Fax: (703) 321-8547

Internet: http://www.ntis.gov/ordering.htm 


\title{
Nonlinear simulation studies of tokamaks and STs
}

\author{
W. Park, J. Breslau, J. Chen, G.Y. Fu, S.C. Jardin, S. Klasky, \\ J. Menard, A. Pletzer, B.C. Stratton, D. Stutman, H.R. Strauss ${ }^{1}$ \\ and L.E. Sugiyama ${ }^{2}$ \\ Princeton University Plasma Physics Laboratory, Princeton, NJ, USA \\ ${ }^{1}$ New York University, NY, USA \\ ${ }^{2}$ Massachusetts Institute of Technology, Cambridge, MA, USA \\ E-mail: wpark@pppl.gov
}

Received 16 October 2002, in final form 5 May 2003

Published 2 June 2003

Online at stacks.iop.org/NF/43/483

\begin{abstract}
The multilevel physics, massively parallel plasma simulation code, M3D has been used to study spherical toris (STs) and tokamaks. The magnitude of outboard shift of density profiles relative to electron temperature profiles seen in NSTX under strong toroidal flow is explained. Internal reconnection events in ST discharges can be classified depending on the crash mechanism, just as in tokamak discharges; a sawtooth crash, disruption due to stochasticity, or high- $\beta$ disruption. Toroidal shear flow can reduce linear growth of internal kink. It has a strong stabilizing effect nonlinearly and causes mode saturation if its profile is maintained, e.g. through a fast momentum source. Normally, however, the flow profile itself flattens during the reconnection process, allowing a complete reconnection to occur. In some cases, the maximum density and pressure spontaneously occur inside the island and cause mode saturation. Gyrokinetic hot particle/MHD hybrid studies of NSTX show the effects of fluid compression on a fast-ion driven $n=1$ mode. MHD studies of recent tokamak experiments with a central current hole indicate that the current clamping is due to sawtooth-like crashes, but with $n=0$.
\end{abstract}

PACS numbers: 52.65.-y, 52.30.-q, 52.30.Cv

\section{Introduction}

The multilevel physics, massively parallel plasma simulation code, M3D [1,2] has been used to study spherical tori (ST) and tokamaks. The effects of strong sheared toroidal flows are included since such flows can be a substantial fraction of the Alfvén speed for STs such as NSTX. Internal reconnection events (IREs) in NSTX are studied and their relation to the sawtooth crash and disruptions in tokamaks are examined. Energetic-particle driven instabilities in NSTX are studied using a hybrid model, and $n=0$ reconnection process is studied to explain current clamping in tokamak experiments with a current hole. M3D currently has physics levels of MHD, two-fluids [3], and a hybrid gyrokinetic-particle/fluid models $[4,5]$. It uses unstructured meshes [6], and the parallel processing structure utilizes MPI and the PETSc framework [7]. The equations and methods used in these studies can be found in [1].

\section{Two-dimensional equilibria with strong sheared toroidal flow}

To explain the measured outboard shift of the plasma density relative to the electron temperature profile in NSTX, time asymptotic numerical two-dimensional steady states are obtained with balanced toroidal momentum, current, density, and pressure sources and sinks. Figure 1 shows steady state density profiles with aspect ratio $A=1.3$ and peak beta $\beta_{0}=30 \% ;(a)$ in MHD, without zeroth order flow, $(b)$ with peak Alfvén Mach number $M=0.2$ in a two-fluid model, (c) with $M=0.8$ in MHD, and (d) $M=0.2$ but with mostly trapped hot particles providing about half of the total pressure. Case $(b)$ is relevant to current experimental conditions, and unlike the density, the shift of the magnetic flux surfaces was very small. A two-fluid case is shown, but the MHD model gave a very similar result, and a hybrid model with parallel pressure $20 \%$ higher than perpendicular pressure also gave a result similar to within the uncertainty of experimental measurements. Thus, the relative density shift index of the MHD model at the magnetic axis, $R \partial \rho / \rho \partial R=2 M^{2} / \beta$ should roughly hold in the experiment. However, the data of Shot 105051 initially gave the right-hand side (the centrifugal force term) only half of what was expected. The missing half turned out to be the centrifugal force of the hot particle slowing down distribution.

With four times the rotation of case $(b)$, figure 1(c) has a maximum density five times larger than the nonrotating case and a small density of only 0.005 over most of the core region, 
showing that core confinement is basically lost. A similar loss of confinement can occur even at $M=0.2$, if the pressure is reduced by 16 times. Such a scenario could occur if a thermal quench happens without a corresponding toroidal momentum quench, e.g. in a disruption.

Figure $1(d)$ shows that if the hot particles were mostly trapped $\left(p_{\perp} \gg p_{\|}\right)$, a much larger outboard shift would occur with a substantial loss of confinement in the core region.

\section{Simulation studies of NSTX IREs}

Although three-dimensional global nonlinear simulations with experimental dissipation parameters of large fusion devices are still beyond current computational capabilities, useful insights on experiments can be gained by simulating discharges under less stiff conditions, e.g. more resistive and viscous plasmas. In some cases, e.g. in low- $\beta$ MHD, the nonlinear evolution goes through similar states as long as the resistive time is long enough compared to the Alfvén time, only the rate of change varying with resistivity. With more complex physics, the instability threshold may also depend on dissipation parameters. Even here, the magnetic topology often evolves through similar states when the plasma is unstable to a global mode, because magnetic field line reconnection must satisfy its own constraints. For the effective Lundquist number of a simulation of an experiment to be realistic, the simulation first has to have sufficient resolution of narrow layer structures in all dimensions. For example, global nonlinear simulations with $S=10^{8}$ that follow only a few harmonics are equivalent to a much lower effective $S$, since narrow reconnection layer structures must be resolved in both radial direction and harmonic space. Second, the simulation has to have sufficient 'physical resolution' of the layer structures, e.g. when layer widths become comparable to or smaller than the ion gyroradius, finite ion gyroradius effects should be included in the physics model. The simulations shown in this section are done with $S$ between $10^{3}$ and $10^{4}$, with magnetic Prandtl number of order one. We could have used higher $S$ values if a small number of very long runs were desired, but to maximize physical insights and ensure the reliability of simulation results, many distinct but similar runs have to be compared.

\subsection{Similarities to sawtooth crash and disruption in tokamaks}

NSTX discharges sometimes end with an IRE with the measured central $q$ value near unity. Numerical simulation of such IREs yielded three different categories, each with close similarity to a corresponding tokamak phenomenon. The first category, as shown in figure 2, is similar to a tokamak sawtooth crash. The hot plasma core is replaced by the colder outer plasma. Such cases are commonly seen in low- $\beta$ plasmas.

When the inversion radius is large or the plasma pressure is increased, a similar sawtooth crash can result in global stochastic magnetic field lines as shown in figure 3. This is a similar phenomenon as a disruption due to stochastic field lines in tokamaks.

When the plasma beta is further increased, the local steepening of pressure driven modes becomes a more common cause of IREs, for example as shown in the pressure profiles of figure 4 , both of which have about $20 \%$ beta. The one on the right has predominantly $m=2$ mode, while the one on the left has more mixed $m$ modes. A similar mechanism of disruption, sometimes called the high- $\beta$ disruption, has been found in tokamaks, both in experiment and theory [8], and has also been found in stellarator simulations when the plasma beta is much higher than the design value [9]. These results indicate that the local steepening of pressure driven modes is a general phenomenon, that can occur in a wide spectrum of plasmas including astrophysical objects. In fact, a high- $\beta$ disruption mechanism has been used to explain solar flares [10].

Since IREs in ST seem to have basically the same mechanism as either the sawtooth crash or disruptions in tokamaks, and scientific knowledge is gained by categorizing depending on the underlying physics, most IREs in STs could be categorized as a sawtooth crash, disruption due to stochasticity, or high- $\beta$ disruption, depending on the crash mechanism. (A disruption process involves a thermal quench sometimes with or sometimes without a current quench.) An ST is after all a very small aspect ratio tokamak, but the smallness of the aspect ratio affects the physical processes and the statistics of occurrence of each type of IRE/disruption can be quite different from in a tokamak.

Figure 5 compares the time history of soft x-ray signals (perturbed part), from experiment and simulation, in a case corresponding to a disruption due to stochasticity. The relative position on the short side corresponds to the vertical detector position. Both show similar global structures of a $m=1$ dominant evolution. However, what the simulation does not explain is the long saturation phase of the mode and the mode locking to the resistive wall prior to the crash. The saturation may be explained by stabilizing effects of strong sheared rotation, the $\omega^{*}$ effect, and energetic-particle effects. To study mode locking, the resistive wall, vacuum region, and external coils should be included in the simulation. All these physics and hardwares are incorporated in the M3D code, and benchmarks and simulation studies are currently being performed. Concerning IREs, only the study of plasma rotation effects has been completed and these are reported in the next section.

\subsection{Effects of sheared toroidal flows}

Linear and nonlinear studies are performed starting from the two-dimensional steady state shown in figure $1(b)$, whose toroidal velocity profile is shown in figure 6 . Toroidal rotation modifies the linear eigenmodes as shown in figure 7 , which compares the potential of the incompressible part of velocity in stationary and rotating equilibrium cases. The mode is shifted inboard and the growth rate is reduced from 0.03 to 0.01 (per Alfvén time) for this case with the peak Alfvén Mach number of $M=0.2$. The mode rotation frequency is 0.13 , which roughly corresponds to the flow rotation frequency of the outboard lobe of the mode.

The nonlinear evolution of this mode shows that the toroidal shear flow is strongly stabilizing nonlinearly. If the momentum source rate were large enough that the toroidal velocity profile of figure 6 is roughly maintained, the mode saturates without going through a complete reconnection 
process. However, with realistic momentum source rate, the toroidal flow profile is flattened and complete reconnection occurs. This is due to the fact that a 'reconnection' process of the toroidal flow itself occurs as shown in figure 8,
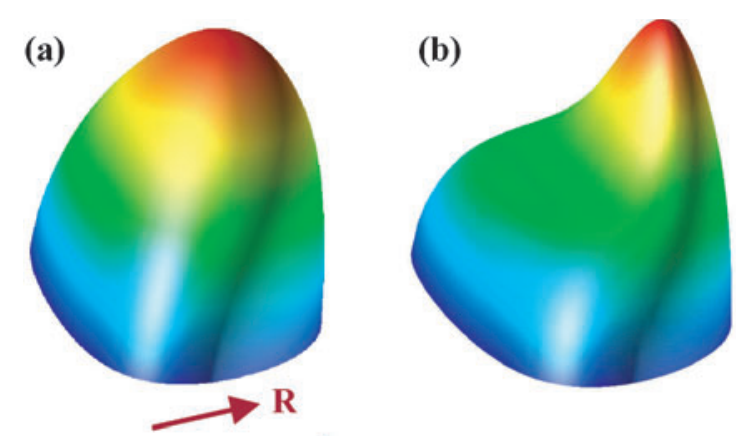

(c)

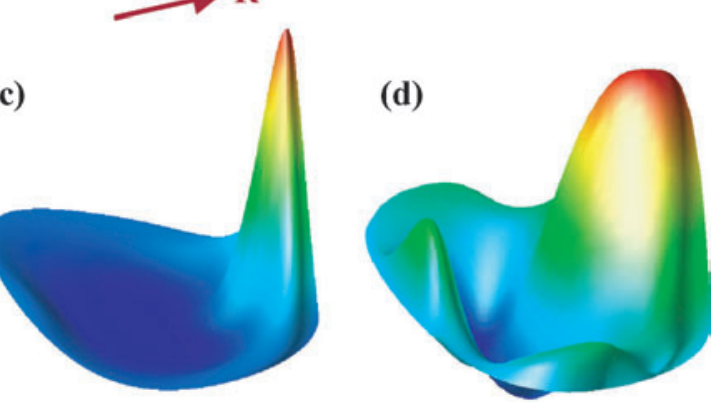

Figure 1. Density profiles of various two-dimensional steady states.

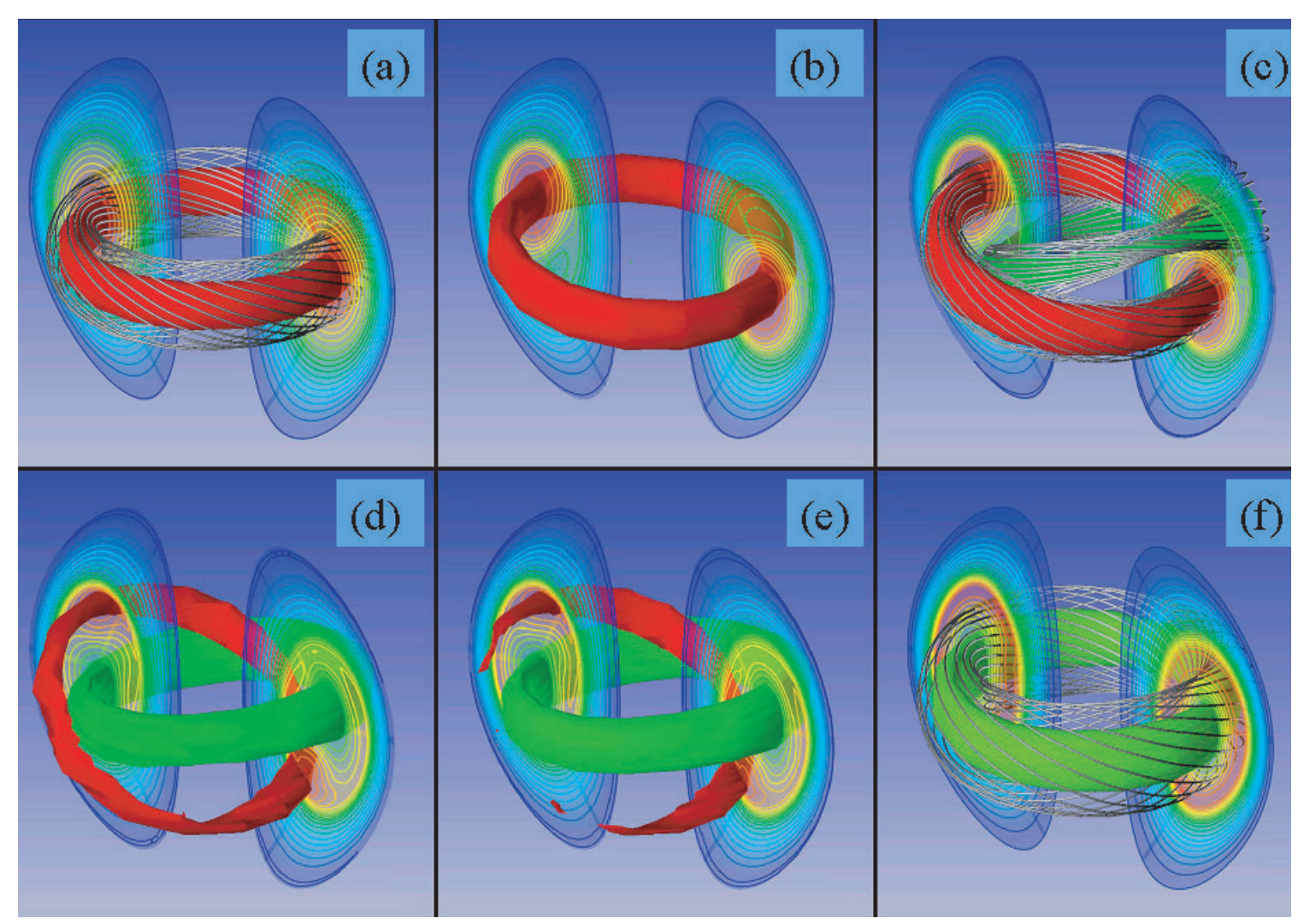

Figure 2. An IRE in a ST simulation which shows a similar behaviour as a tokamak sawtooth crash. Temperature iso-surfaces and contours are shown together with selected magnetic field lines. The hot plasma core is replaced by cold peripheral plasma through reconnecion of field lines. which compares pressure and toroidal flow profiles during the reconnection process. The two profiles are similar, because both evolve under conservation laws, of thermal energy and angular momentum, respectively, inside the reconnected flux bundles. The upper regions are flattened due to the existence of a large $m=1$ magnetic island there. Such flattening of both pressure and toroidal flow have been observed in experiments in the presence of a large magnetic island [11]. At the complete reconnection, both pressure and toroidal flow patterns are flattened. Thus $m=1$ reconnection normally goes through to completion in the MHD model by flattening the toroidal flow profile with it.

If the plasma survives a crash, sometimes a subsequent reconnection process can saturate by spontaneously generating a curious profile shown in figures 9 and 10. The temperature is highest in the original 'hot' spot, but the density and pressure peak inside the island, probably due to the centrifugal force effect on density. Such a profile has been known to be stabilizing to a $m=1$ reconnection process, and leads to a saturated state [12]. The driving free energy of the usual $m=1$ reconnection comes partly from the outward shift of the central high pressure region inside the $q=1$ surface, while in the present case such a shift would not be energetically favourable because the pressure in the island which would be moving inward is the highest. (A similar mechanism can also explain the 'snake' phenomenon in tokamaks, as will be reported later.)

Thus, MHD physics with shear flow could explain some of $m=1$ saturation cases seen in NSTX. At the least, such 

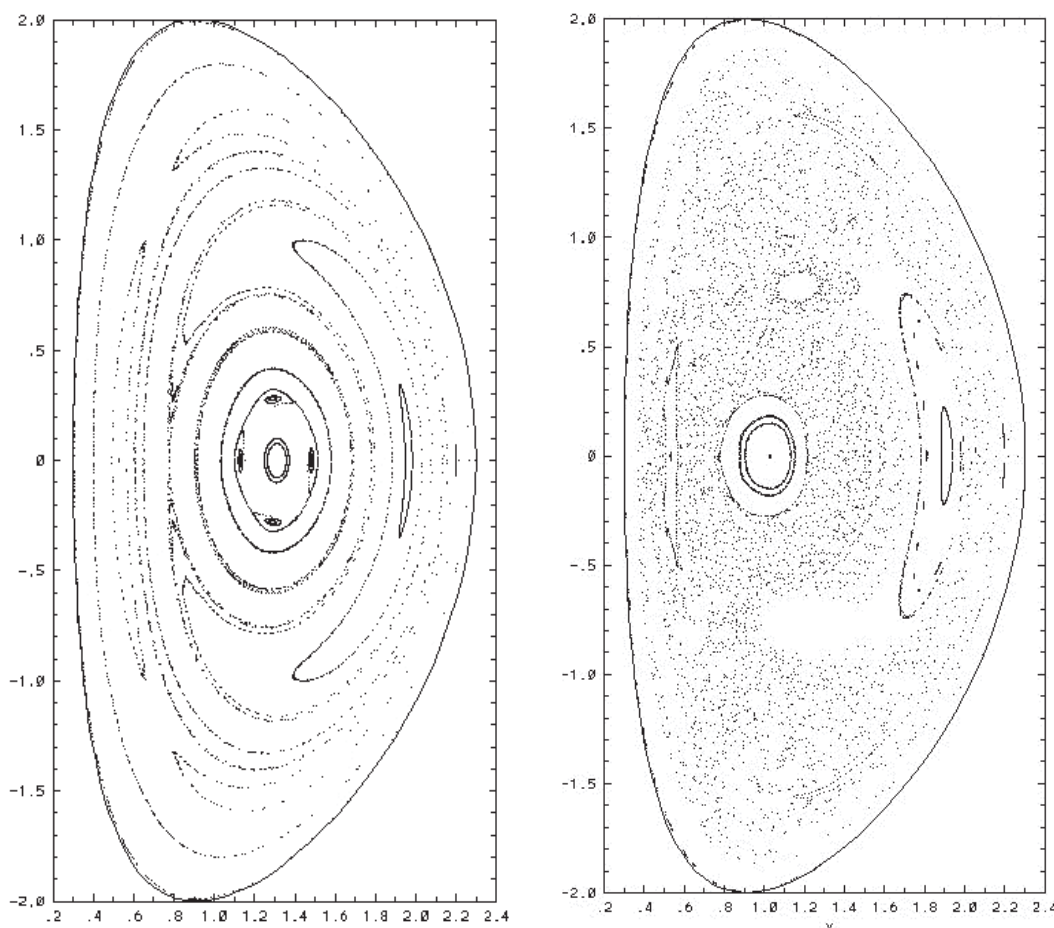

Figure 3. Puncture plots of field lines show that magnetic islands overlap and become stochastic.
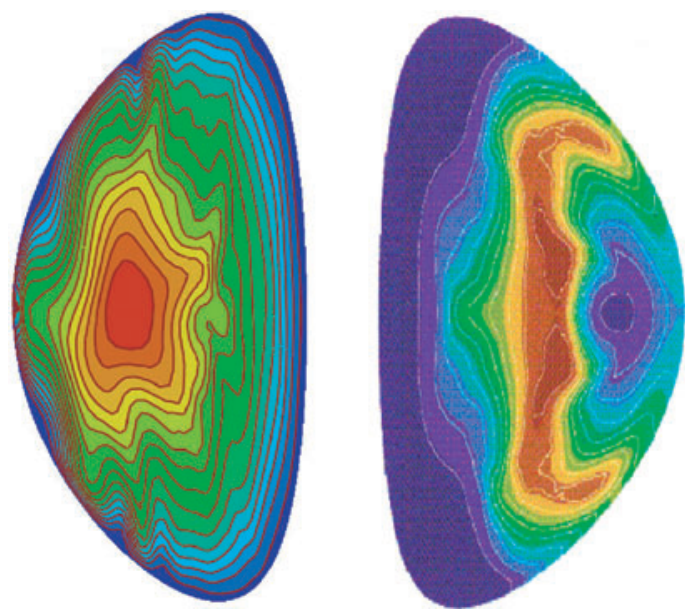

Figure 4. IREs in high- $\beta$ ST simulation show similar behaviour as tokamak high- $\beta$ disruptions.

effects would contribute with additional $\omega^{*}$ and energeticparticle stabilization effects to eventually cause the saturation of the $m=1$ mode. Such studies are currently being carried out using the two-fluid and hybrid particle/MHD levels of the code.

\section{Hot particle/MHD hybrid studies of energetic ion driven modes}

Energetic-particle driven MHD modes are studied for ST plasmas using the hybrid model in M3D $[4,5]$. In the hybrid model, the plasma is divided into the bulk plasma and an energetic-particle component. The bulk plasma, which includes thermal electrons and ions, is treated as a single

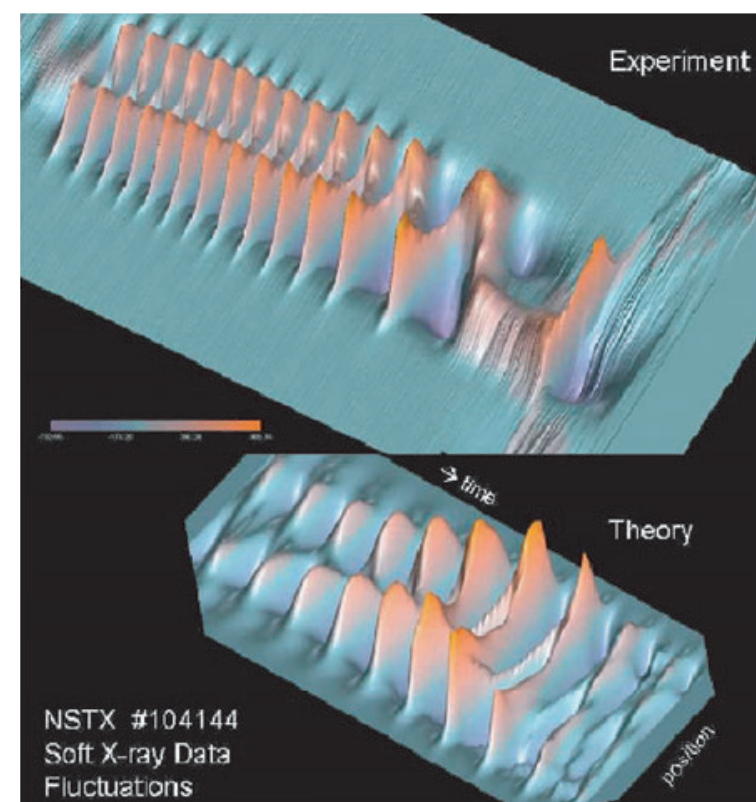

Figure 5. Soft x-ray data are compared between experiment and theory.

fluid and the energetic-particles are described by gyrokineticparticles. The effects of hot particles enter through the momentum equation via the hot particle stress tensor (pressure coupling). The $\delta f$ method is used in calculating the stress tensor from the hot particles which are advanced in a selfconsistent electromagnetic field.

We consider parameters and profiles similar to the NBI-heated NSTX plasmas: aspect ratio $A=1.3$, elongation $\kappa=2.0$, triangularity $\delta=0.3$, central and edge safety factor 


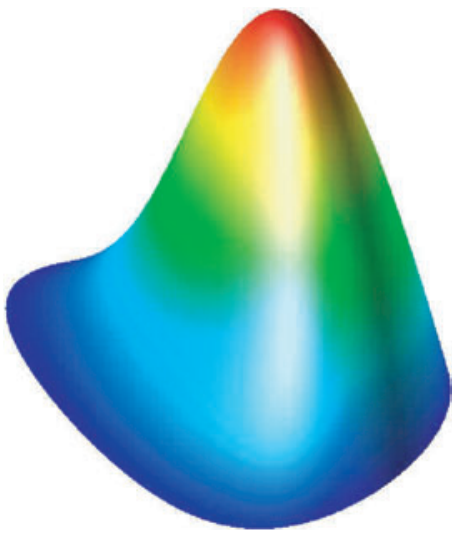

Figure 6. Toroidal velocity profile of two-dimensional steady state.
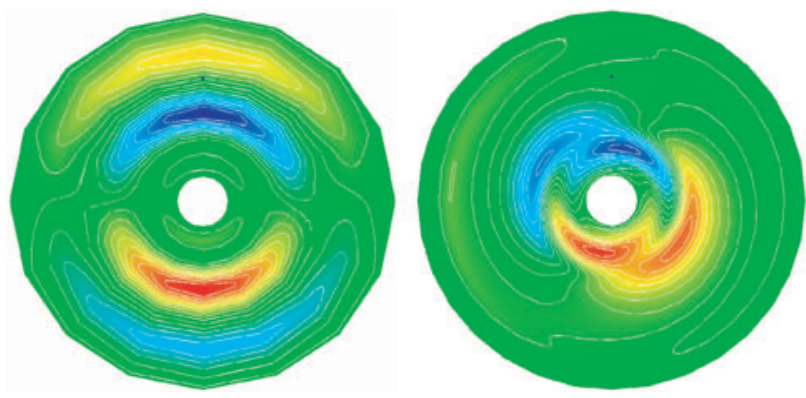

Figure 7. Velocity potential (incompressible part) profiles of $n=1$ linear eigenmode on the midplane of torus. Cases without flow and with flow $M=0.2$ are compared.
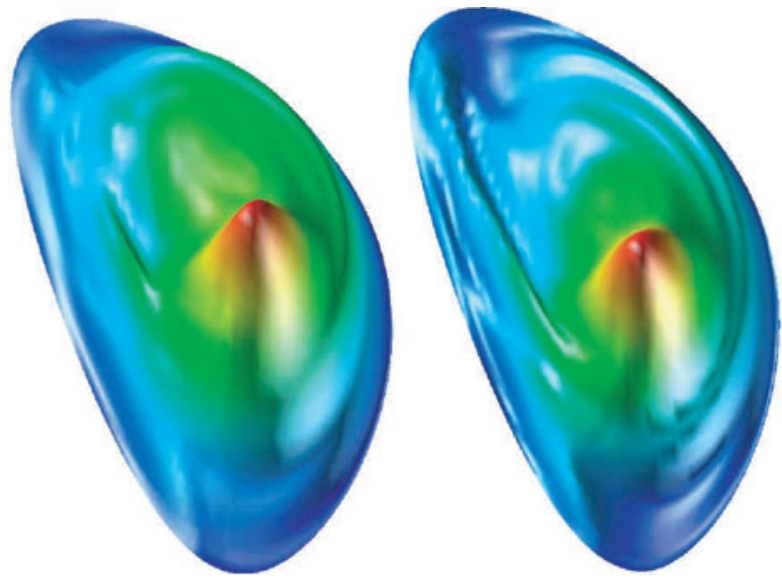

Figure 8. Pressure profile and toroidal velocity profile show close similarities. A large $m=1$ island is located at the upper region.

$q(0)=1.4$ and $q(a)=7.3$, ratio of hot ion speed and Alfvén speed is 3.9 , and the normalized hot ion gyroradius, $\rho_{\mathrm{h}} / a=$ 0.09 . Figure 11 shows a linearly unstable TAE-like eigenmode with its mode frequency comparable to the expected TAE frequency at the $q=1.5$ surface. This unstable TAE is driven by hot ions at hot ion beta value of $\beta_{\mathrm{h}}=3.8 \%$ and zero bulk plasma beta. However, when the bulk plasma beta is increased to $\beta_{\text {bulk }}=24.5 \%$, the mode frequency is reduced by a factor of 5 to $\omega=0.07 v_{\mathrm{A}}(0) / R$. The corresponding eigenmode structure is shown in figure 11 on the right. The frequency of this new mode is located below the beta-induced continuum gap near the centre of the plasma and thus it may be called
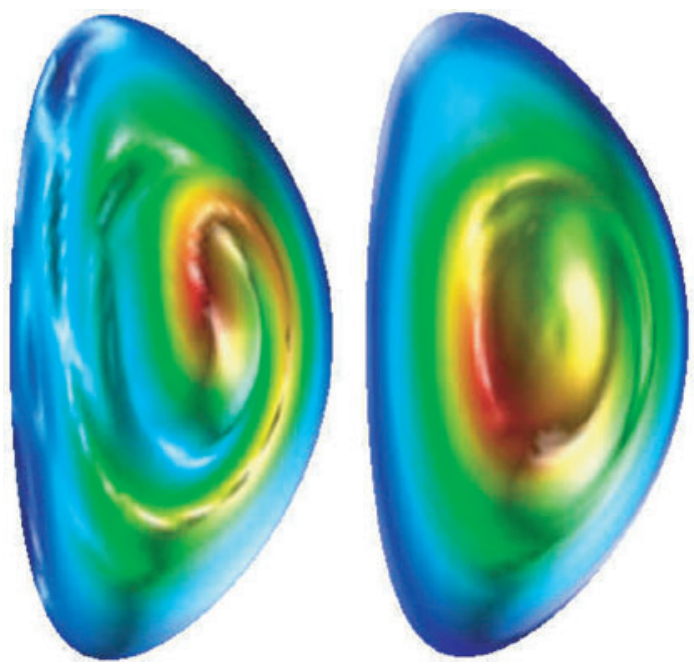

Figure 9. Density and temperature profiles, with density (and pressure) peak inside the island and temperature peak inside the original hot spot.

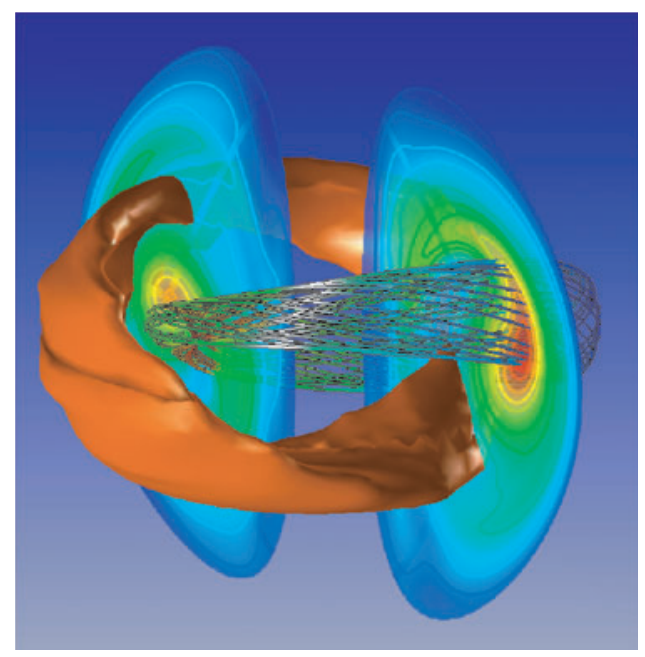

Figure 10. A saturated state. A temperature iso-surface, density contours, and a field line are shown.

beta-induced Alfvén eigenmode (BAE). To test whether this mode is indeed beta driven, the ratio of the specific heat, $\Gamma$, is changed to zero (i.e. zero sound speed) from $\Gamma=\frac{5}{3}$, and the mode has reverted to a TAE mode. The mode frequency is higher than $\omega^{*}$ of bulk ions. The stabilizing effect of bulk ion Landau damping on this BAE mode is currently being studied using the ion-gyrokinetic-particles/electron-fluid hybrid level of M3D code.

\section{Interpretation of tokamak experiments with a current hole}

Recent experiments in JET and JT-60 with a fast current ramp-up and external current drive exhibit a central region with virtually zero current density $[13,14]$. Simulation of these current hole discharges indicate that the current clamping is due to sawtooth-like crashes, but with toroidal mode number $n=0$, rather than $n=1$. Figure 12 shows the poloidal flux contours during one such crash, using Lundquist number $S=10^{4}$. 

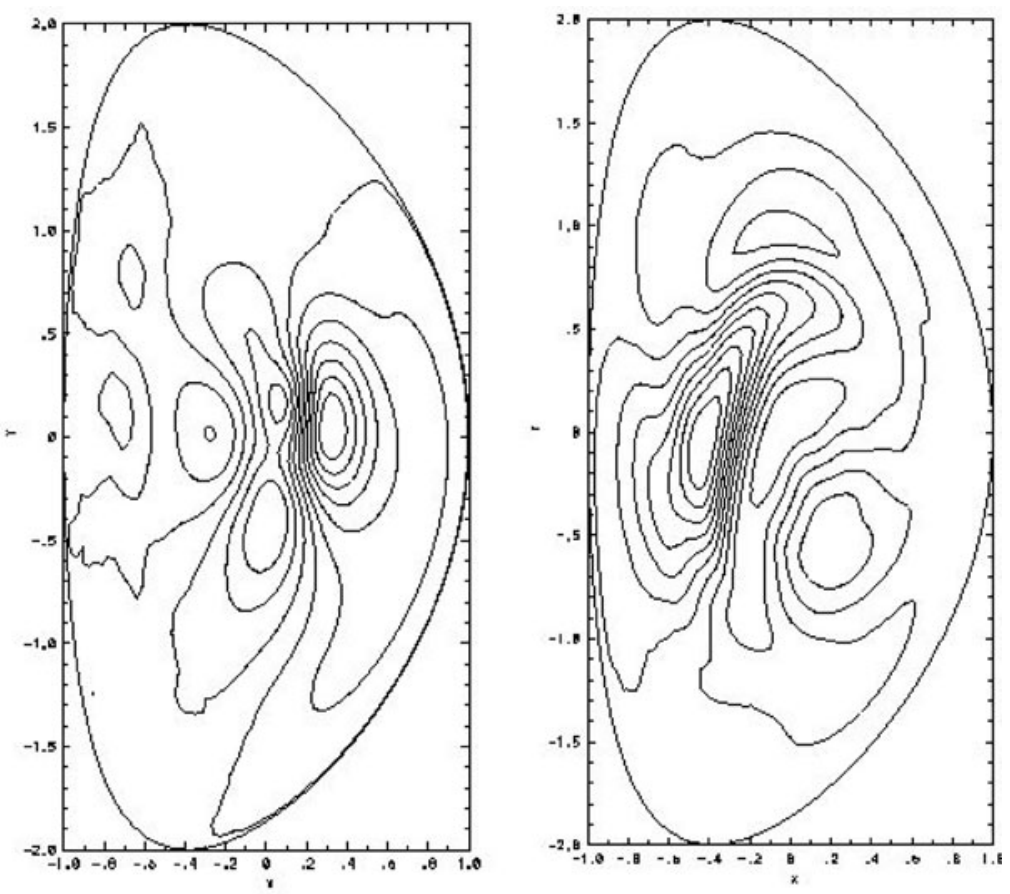

Figure 11. TAE mode on the left. BAE mode on the right is destabilized at higher $\beta$.
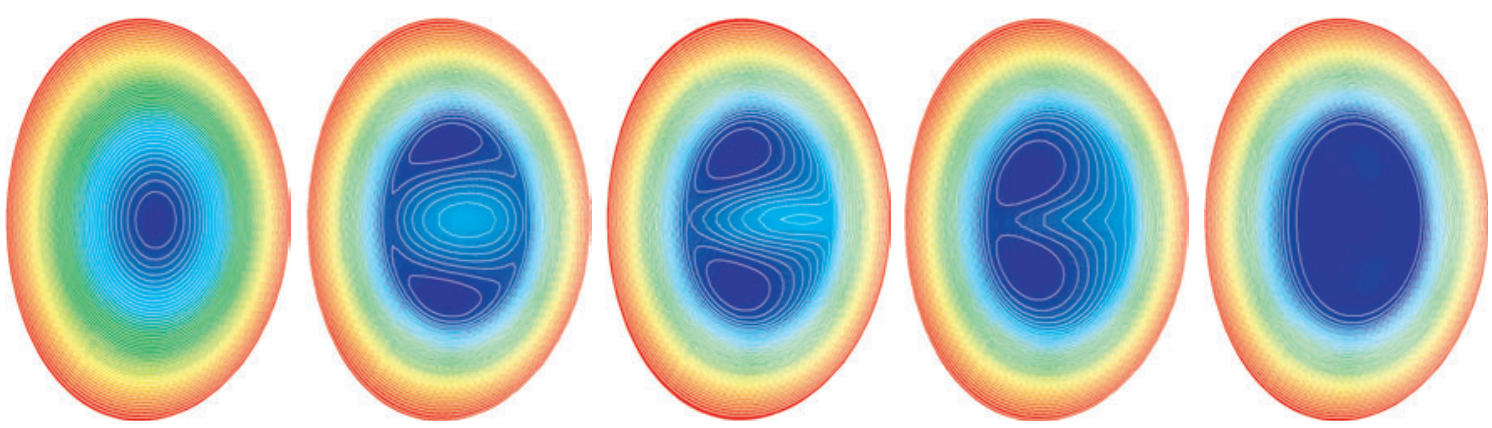

Figure 12. Time sequence of poloidal flux contours during a $n=0$ reconnection process which clamps core current near zero.

(Rationale for using smaller $S$ is given in section 3.) It shows a complete reconnection with predominant $m=1$ mixed with $m=2$. The crash free energy comes from two sources; (1) the two-dimensional state has no regular equilibrium and gives rise to a singular current sheet with finite jump of magnetic field strength (related to the Shafranov shift), and (2) a free energy analogous to the usual $n=1$ internal kink. Figure 13 shows the time history of the toroidal current density across the horizontal midplane, clearly revealing the clamping of the core current density to near zero. This simulation includes a general three-dimensional perturbation, and shows that some three-dimensional modes are also unstable. The implications of such modes are currently being studied.

The above simulations have used negligibly small plasma beta. For plasmas with significant $\beta$, MHD simulations tend to give saturation of the $n=0$ internal kink, thus allowing the central current density to reach significantly negative values. However, such a saturated state does not last in a more accurate two-fluid model, and the net effect results in evolutions similar to what are presented above. The details of these results will be reported later.

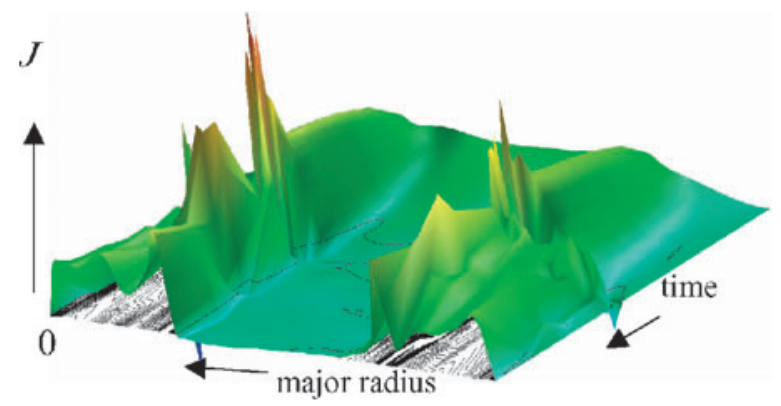

Figure 13. Time history of toroidal current density, showing multiple $n=0$ reconnections, and some three-dimensional instability.

\section{Conclusions}

Various physics levels of the M3D code are used to study ST and tokamak phenomena. The magnitude of the outboard shift of density profile relative to electron temperature profile seen in NSTX under strong toroidal flow is explained. IREs 
in ST discharges can be classified depending on the crash mechanism, just as in tokamak discharges - sawtooth crash, disruption due to stochasticity, or high- $\beta$ disruption. Toroidal shear flow can reduce the linear growth of the internal kink. It has strong stabilizing effects nonlinearly and can cause mode saturation if its profile is maintained, e.g. through a fast momentum source. Normally however, the flow profile itself flattens during the reconnection process, allowing a complete reconnection. In some instances, the maximum density and pressure spontaneously occur inside the island and cause a mode saturation. Hot particle/MHD hybrid simulation of NSTX has produced a BAE mode. Finally, the current clamping in tokamak experiment with a current hole is explained as due to an $n=0$ sawteeth reconnection process. Even though much less stiff cases than the actual experimental parameters are used in the studies presented here, many useful insights are gained. Future simulation studies will have increasingly realistic parameters with appropriate physical and numerical resolutions for the parameters used.

\section{Acknowledgments}

This work was supported by the United States Department of Energy under Contracts DE-AC02-76-CHO-3073, DE-FG02-91ER54109, and DE-FG02-86ER53223.

\section{References}

[1] Park W. et al 1999 Plasma simulation studies using multilevel physics models Phys. Plasmas 61796
[2] Strauss H.R. and Park W. 1998 MHD effects on pellet injection in tokamaks Phys. Plasmas 52676

[3] Sugiyama L.E. and Park W. 2000 A nonlinear two-fluid model for toroidal plasmas Phys. Plasmas 74644

[4] Park W. et al 1992 Three dimensional hybrid gyrokinetic-MHD simulation Phys. Fluids B 42033

[5] Fu G.Y. and Park W. 1995 Nonlinear hybrid simulation of the toroidicity-induced Alfvén eigenmode Phys. Rev. Lett. 741594

[6] Strauss H.R. and Longcope D.W. 1998 J. Comp. Phys. 2318

[7] Balay S., Gropp W.D., McInnes L.C. and Smith B.F. 1997 Efficient management of parallelism in object oriented numerical software libraries Modern Software Tools in Scientific Computing ed E. Arge et al (Basel, Switzerland: Birkhauser Press)

[8] Park W. et al 1995 High- $\beta$ disruption in tokamaks Phys. Rev. Lett. 751763

[9] Strauss H.R. et al 2002 Nonlinear MHD and energetic particle modes in stellarators Nucl. Fusion submitted

[10] Shibasaki K. 2001 High-beta disruption in the solar atmosphere Astrophys. J. 557326

[11] Menard J. et al 2003 Beta-limiting MHD instabilities in improved performance NSTX spherical torus plasmas $\mathrm{Nucl}$. Fusion $\mathbf{4 3} 330$

[12] Park W., Monticello D.A. and Chu T.K. 1987 Sawtooth stabilization through island pressure enhancement Phys. Fluids 30285

[13] Hawkes N.C. et al 2001 Observation of zero current density in the core of JET discharges with lower hybrid heating and current drive Phys. Rev. Lett. 87115001

[14] Stratton B.C. et al 2002 The role of axisymmetric reconnection events in JET discharges with extreme shear reversal Plsma Phys. Control. Fusion 441127 


\section{External Distribution}

Plasma Research Laboratory, Australian National University, Australia

Professor I.R. Jones, Flinders University, Australia

Professor João Canalle, Instituto de Fisica DEQ/IF - UERJ, Brazil

Mr. Gerson O. Ludwig, Instituto Nacional de Pesquisas, Brazil

Dr. P.H. Sakanaka, Instituto Fisica, Brazil

The Librarian, Culham Laboratory, England

Mrs. S.A. Hutchinson, JET Library, England

Professor M.N. Bussac, Ecole Polytechnique, France

Librarian, Max-Planck-Institut für Plasmaphysik, Germany

Jolan Moldvai, Reports Library, Hungarian Academy of Sciences, Central Research Institute for Physics, Hungary

Dr. P. Kaw, Institute for Plasma Research, India

Ms. P.J. Pathak, Librarian, Institute for Plasma Research, India

Ms. Clelia De Palo, Associazione EURATOM-ENEA, Italy

Dr. G. Grosso, Instituto di Fisica del Plasma, Italy

Librarian, Naka Fusion Research Establishment, JAERI, Japan

Library, Laboratory for Complex Energy Processes, Institute for Advanced Study, Kyoto University, Japan

Research Information Center, National Institute for Fusion Science, Japan

Dr. O. Mitarai, Kyushu Tokai University, Japan

Dr. Jiangang Li, Institute of Plasma Physics, Chinese Academy of Sciences, People's Republic of China

Professor Yuping Huo, School of Physical Science and Technology, People's Republic of China

Library, Academia Sinica, Institute of Plasma Physics, People's Republic of China

Librarian, Institute of Physics, Chinese Academy of Sciences, People's Republic of China

Dr. S. Mirnov, TRINITI, Troitsk, Russian Federation, Russia

Dr. V.S. Strelkov, Kurchatov Institute, Russian Federation, Russia

Professor Peter Lukac, Katedra Fyziky Plazmy MFF UK, Mlynska dolina F-2, Komenskeho Univerzita, SK-842 15 Bratislava, Slovakia

Dr. G.S. Lee, Korea Basic Science Institute, South Korea

Institute for Plasma Research, University of Maryland, USA

Librarian, Fusion Energy Division, Oak Ridge National Laboratory, USA

Librarian, Institute of Fusion Studies, University of Texas, USA

Librarian, Magnetic Fusion Program, Lawrence Livermore National Laboratory, USA

Library, General Atomics, USA

Plasma Physics Group, Fusion Energy Research Program, University of California at San Diego, USA

Plasma Physics Library, Columbia University, USA

Alkesh Punjabi, Center for Fusion Research and Training, Hampton University, USA

Dr. W.M. Stacey, Fusion Research Center, Georgia Institute of Technology, USA

Dr. John Willis, U.S. Department of Energy, Office of Fusion Energy Sciences, USA

Mr. Paul H. Wright, Indianapolis, Indiana, USA 
The Princeton Plasma Physics Laboratory is operated by Princeton University under contract with the U.S. Department of Energy.

\author{
Information Services \\ Princeton Plasma Physics Laboratory \\ P.O. Box 451 \\ Princeton, NJ 08543
}

Phone: 609-243-2750

Fax: 609-243-2751

e-mail: pppl_info@pppl.gov

Internet Address: http://www.pppl.gov 